Pathology, Hallym University Sacred-Heart Hospital, Hallym University College of Medicine, Anyang, Korea

Background: The cribriform-morular variant of papillary thyroid carcinoma (CMV-PTC) is an unusual neoplasm with an important association with familial adenomatous polyposis in young women, characterized by a peculiar histology with mixed cribriform, papillary, solid, tall columnar, and morular patterns. However, it can also occur sporadically. The molecular pathogenesis of sporadic CMV-PTC is not completely understood. Here, we report cases of three patients with sporadic CMV-PTC with PIK3CA mutations.

Methods: Using sequencing analyses and immunohistochemistry, we examined KRAS, BRAF, PIK3CA, and CTNNB1 mutations and related proteins, including $\beta$-catenin, PTEN, CD10, estrogen receptor (ER), progesterone receptor (PR), cytokeratin 19, and cyclin D1 in three CMV-PTCs.

Results: The three patients were teen-aged women. The tumors were solitary and encapsulated without nodal metastasis. They showed no recurrence for more than six years following the operation. Three tumors were diffusely positive for $\beta$-catenin, cyclin D1, and PTEN. The biphasic immunohistochemical patterns between the morular and non-morular components were identified; the non-morular components were positive for ER, PR, and cytokeratin 19, whereas the morular components showed CD10 positivity. All tumors harbored the same mutation in exon 9, codon 545 of the PIK3CA gene (E545A), whereas the KRAS, BRAF, and CTNNB1 mutations were not detected.

Conclusions: This is the first study identifying the PIK3CA mutation specifically in sporadic CMV-PTC. The presence of the PIK3CA mutation and the wild-type KRAS, BRAF, CTNNB1 genes, and the intact PTEN expression in 3 sporadic CMV-PTCs may suggest the possible contribution of the PIK3CA mutation in its tumorigenesis.

\section{DIAGNOSTIC ROLE OF IMMUNOCYTOCHEMISTRY IN MALIGNANT PLEURAL MESOTHELIOMA}

Lam Nguyen Son ${ }^{1}$, Thanh Tran Dinh ${ }^{2}$, Dung Nguyen Huy ${ }^{3}$ ${ }^{1}$ Head of Pathology Department of Pham Ngoc Thach Hospital, Ho Chi Minh City, ${ }^{2} \mathrm{Head}$ of Oncology Department of Pham Ngoc Thach Hospital, and ${ }^{3}$ Director of Pham Ngoc Thach Hospital, Vietnam

Background: Malignant pleural mesothelioma is a difficult diagnosis, more severe cases may not perform by pleural biopsy. With these cases, the technique of immunocytochemistry was performed proposals.

Methods: A prospective, cross-sectional descriptive statistics with clinical series cases.

Results:

- Implementing 58 cases of malignant pleural mesothelioma by immunocytochemistry

- Technical Cytospin method has helped for immunocytochemistry have high results

- Should be done more staining with markers for the other diagnostic diseases: lung cancer and non-lung cancers that metastatic to pleura

- The results of the data:

- Sensitivity: $84.06 \%$

- Specificity: $100 \%$
- Positive Prognostic Value: $100 \%$

- Negative Prognostic Value: $92.25 \%$

- Accuracy $94.12 \%$

Conclusions: The diagnosis of malignant pleural mesothelioma is always a difficult diagnosis. Needing the differential diagnosis with other types of diseases, such as lung cancer and non-lung cancers that to pleural pulmonary metastases. Staining with immunocytochemistry in cases of pleural effusion showed relatively high value. This suggests that the ability to diagnose initially screening properties of this technique is very good, especially valuable in the diagnosis of severe disease progression cannot perform other diagnostic techniques.

References

1. Henderson D, Cagle P. Pleural Pathology. Pathology Int 2004; 54 (Suppl 1): S490-S505.

2. Metzgeroth G, Kuhn C, Schultheis B, et al. Diagnostic accuracy of cytology and immunocytology in carcinomatous effusions. Cytopathology 2008; 19: 205-11.

3. Motherby H, Nadjari B, Friegel P, et al. Diagnostic accuracy of effusion cytology. Diagn Cytopathol 1999; 20: 350-61.

4. Ikeda K, Tate G, Suzuki T, et al. Comparison of immunocytochemical sensitivity between formalin-fixed and alcoholfixed specimens reveals the diagnostic value of alcohol-fixed cytocentrifuged preparations in malignant effusion cytology. Am J Clin Pathol 2011; 136: 934-42.

5. Kouki Inai. Pathological Diagnosis of Asbestos-exposurerelated Diseases and Pathological Diagnosis of Mesothelioma. Training Course on Diagnosis of Asbestos Related Diseases, 2010: 7-10, 5-9.

\section{ADENOMATOID TUMOUR OF TUNICA ALBUGINEA OF TESTIS}

Tee Lang, Frank Lee

Khoo Teck Puat Hospital, Singapore

Background: Adenomatoid tumours are benign mesothelial neoplasm of paratesticular area and they commonly occur in the epipidymis. Uncommonly, adenomatoid tumours can arise from the tunica and present as intratesticular mass.

Aim: To describe a case of adenomatoid tumour presented as intratesticular tumour and to review its ultrasonographic appearance and histological features.

Method: A 39-year-old Sri Lankan man presented himself to the emergency department with acute onset of abdominal pain radiated to right groin. There was no obvious mass in the abdomen or groin. Serum tumour markers including $\beta$-HCG, $\alpha$ fetoprotein and lactate dehydrogenase were normal. An ultrasound scan identified a $10 \mathrm{~mm}$ hypoechoic solid nodule with minimal vascularity in the lower pole of right testis. The patient opted for conservative management but a follow- up ultrasound scan two months later showed a slight increased in size. An elective orchidectomy was performed to remove the nodule.

Result: An orchidectomy specimen with testis measured $40 \times 28 \times 20 \mathrm{~mm}$. The cut surface showed a firm yellow nodule at the lower pole measured $9 \times 5 \mathrm{~mm}$. The nodule had a well circumscribed border but lacked a capsule and it appeared to arise from the tunica albuginea abutted into the testicular parenchyma. Microscopically, the tumour showed an infiltrating growth pattern with cords and strands of oval tumour cells with eosinophilic cytoplasm within fibrous tissue. Occasional tumour 
cells with vacuolated cytoplasm were noted. Immunohistochemistry performed on tumour showed positive staining with calretinin, WT1, CAM5.2 and negative staining with Berep4, CD34, and S100.

Conclusion: Adenomatoid tumours mimicking testicular tumour can present a challenge for urologist to decide on the most appropriate surgery. Clinical awareness of the unusual location of adenomatoid tumour could elect patient for testis sparing surgery.

\section{EPITHELIAL SPARC EXPRESSION IS CORRELATED WITH POOR SURVIVAL AS WELL AS MMP-2 EXPRESSION OF INVASIVE BREAST CARCINOMA}

Ji Shin Lee, Ga-Eon Kim, Jae Hyuk Lee, Jong Hee Nam, Chan Choi

Department of Pathology, Chonnam National University Medical School, Gwangju, Republic of Korea

Background: Invasion and metastasis are the direct causes of mortality in breast cancer patients. Secreted protein acidic and rich in cysteine (SPARC) plays a crucial role in the process of tumor invasion and metastasis in some tumors. Matrix metalloproteinases (MMPs) degrade the extracellular matrix and participate in tumor invasion and metastasis.

Aims: The aims were to assess the role of SPARC alteration in the breast carcinogenesis and its association with outcome variables and MMP expression in invasive breast carcinoma (IBC). Methods: Immunohistochemical staining with anti-SPARC antibody was performed in a total of 300 patients with 26 normal breasts, 76 ductal carcinoma in situ (DCIS), and 198 IBC using tissue microarray. In addition, we also performed immunohistochemical staining for MMP-2 and MMP-9 in IBC.

Results: Epithelial SPARC expression appeared to increase progressively along the continuum of neoplastic changes from normal breast epithelium to IBC $(\mathrm{P}<0.001)$. The patients with high epithelial SPARC expression had a significantly poorer prognosis for disease-free and overall survival than those with low epithelial expression ( $p=0.002$ and $p=0.048$, respectively). Epithelial SPARC expression was independently associated with an increased risk for poor disease-free survival. Epithelial SPARC expression was statistically correlated with MMP-2 expression $(p<0.05)$.

Conclusions: Tumor progression in breast epithelium is accompanied by increased epithelial SPARC expression. High epithelial SPARC expression may serve as a new parameter for the prognostic prediction in patients with IBC. SPARC-mediated degradation of the extracellular matrix, and its possible association with MMP-2, may contribute to the progression of IBC.

\section{HEPATIC REACTIVE LYMPHOID HYPERPLASIA ASSOCIATED WITH MALIGNANT TUMOR}

Sik Lee, Jun Sang Bae, Woo Sung Moon

Department of Pathology, Internal Medicine, Chonbuk National University, Medical School, and Research Institute for

Endocrine Sciences, Jeonju, Korea
Reactive lymphoid hyperplasia (RLH) is a rare benign condition that forms a mass-like lesion characterized by proliferation of non-neoplastic lymphocytes forming follicles and germinal centers. RLH is thought to represent a reactive immunological response, and some cases may arise in association with malignancy. Herein, we present four cases of hepatic RLH associated with malignant tumor clinically mimic metastatic tumor. All 4 patients were women ranging from 45 to 74 years (mean, 59 years). The hepatic lesions ranged from 0.5 to $1.5 \mathrm{~cm}$. Radiologically, these lesions mimic a primary or metastatic malignancy. All 4 lesions were associated with internal malignancies; renal cell carcinoma, ovarian mucinous carcinoma, colon adenocarcinoma and bile duct carcinoma, respectively. Microscopically, the lesions are characterized by formation of follicles with polymorphic and polyclonal cell population and active germinal centers. There was no evidence of lymphoepithelial lesion or monoclonal B-cell proliferation. It can be suggested that the pathogenesis of RLH of the liver in patients with malignant tumor may be related to an immunologic abnormality that is caused by the malignant tumor itself or previous surgery for the tumors.

\section{SELECTION OF SUPER-INVASIVE CELL POPULATION FROM THE GLIOBLASTOMA CELL LINE AND ANALYSIS OF INVASION-ASSOCIATED BIOMARKERS}

Kyu Sang Lee, Hyun Sook Jung, Seyoung Moon, Gheeyoung Choe

Department of Pathology, Seoul National University Bundang Hospital, Seoul National University College of Medicine, Korea

Background: Glioblastoma is characterized by aggressive local invasiveness, making complete surgical resection nearly impossible. To identify mechanisms of glioblastoma invasion, we isolated a subpopulation of more invasive cells and evaluated the expression of invasion related factors including integrin subunits, matrix metalloproteinase-2 (MMP2), fascin, actin and Erk1/2.

Methods: We selected the first $10 \%$ of invading cells (U87-Inv) from U87MG using laminin-2 (merosin) coated Transwell filters. To characterize the more invasive cells, we used wound assay, gelatin zymography, proliferation assay, RT-PCR for integrinreceptors for merosin, western blot and immunofluorescence for fascin and actin.

Results: Migration rate of U87-Inv cells exhibited about $20 \%$ increase compared with relatively lower invasive cells (U87Non). U87-Inv cells demonstrated faster wound healing, whereas lower proliferative activity. The expression levels of integrin $\alpha 1$, $\alpha 7$, and $\beta 1$ increased a little higher (about 1.2-fold for $\beta 1$ and 1.5 -fold for $\alpha 1$ and $\alpha 7$ ) in U87-Inv compared with U87-Non, while the expression of integrin $\alpha 6$ and $\beta 4$ reduced in U87-Inv (about 0.4 -fold for $\beta 4$ and 0.6-fold for $\alpha 6$ ). U87-Inv cells revealed increase of fascin, actin and MMP2, whereas they showed decrease of phosphorylated Erk1/2. By immunofluorescence study, U87-Inv cells revealed extensive lamellipodia expressing both fascin and actin. 